\title{
ON THE ANALYTIC FUNCTION WHICH APPEARS IN KRONECKER'S LIMIT FORMULA FOR CM-FIELDS
}

\author{
TOSHIAKI SUZUKI
}

Hecke stated in [1] Kronecker's limit formula for CM-fields without proof, in which "Die zu $\log \eta(z)$ analog Funktion" appears, and he investigated in [3] the behaviors of this function under modular substitutions. S. Konno also discussed Kronecker's limit formula for CM-fields in [4].

In this paper we shall investigate "an analytic function analogous to $\log \left[\vartheta_{11}(a z+b / N, z) / \eta(z)\right]$ " which appears in the second limit formula for CM-fields. We make use of Hecke's method of [3] and the functional equation of partial zeta functions in order to obtain the behaviors of our analytic function under modular substitutions. We deal with them in prime levels, but this restriction is not essential.

The author would like to thank the referee who read the manuscript very carefully and advised on several points.

\section{§1. Limit formula}

Let $K$ be a real quadratic number field which has class number one, $\vartheta$ the integer ring of $K$ and $(\delta)$ its different. Let $f$ be a prime number of $\vartheta$ and $E_{\mathrm{f}}^{+}$be the group of totally positive units $\eta$ such that $\eta \equiv 1(\mathfrak{f})$. For $c, c^{\prime} \in \vartheta, c^{\prime}$ is called $E_{j}^{+}$-associated with $c$ if $c^{\prime}=\eta c$ for some $\eta \in E_{j}^{+}$. If $c \in \vartheta(c \neq 0)$, we choose a fixed representative $(c)_{i}^{+}$from the class of $E_{\ddagger}^{+}-$ associated numbers with $c$. For $c, c^{\prime}, d, d^{\prime} \in \vartheta$, a pair $\left(c^{\prime}, d^{\prime}\right)$ is called $E_{\mathrm{f}}^{+}$associated with $(c, d)$ if both relations $c^{\prime}=\eta c$ and $d^{\prime}=\eta d$ hold for some $\eta \in E_{\mathrm{f}}^{+}$. If $(c, d) \neq(0,0)$, we also choose a fixed representative pair $(c, d)_{\mathrm{f}}^{+}$ from the class of $E_{\mathrm{f}}^{+}$-associated pairs with $(c, d)$.

As usual, $K$ is embedded into $\boldsymbol{R}^{2}$ by $c \mapsto\left(c_{1}, c_{2}\right)$ where $c_{1}=c$ and $c_{2}$ is the conjugate of $c$. Let $H^{2}$ denote the product of two copies of the complex upper half plane $H$. For $z=\left(z_{1}, z_{2}\right) \in H^{2}$ or $\in(C-R)^{2}$ and $c, d \in K$, we set:

Received January 20, 1983.

Revised September 28, 1984. 


$$
\begin{aligned}
& c z+d=\left(c_{1} z_{1}+d_{1}, c_{2} z_{2}+d_{2}\right) \in(C-R)^{2} ; \\
& N(z)=z_{1} z_{2}, N(c)=c_{1} c_{2} ; S(z)=z_{1}+z_{2}, S(c)=c_{1}+c_{2} .
\end{aligned}
$$

We put $D=|N(\delta)|$ and $f=|N(\mathfrak{f})|$.

We consider a generalization of Kronecker's double series. For $\alpha, \beta$ $\in \vartheta($ not simultaneously congruent to $0 \bmod \mathfrak{f}), s \in C$ and $z \in H^{2}$, we define

$$
\begin{aligned}
G(z ; s) & =G(z ; \alpha, \beta ; \mathfrak{f} ; s) \\
& =\sum_{(c, d)_{f}^{+}} \exp \left(2 \pi i S\left(\frac{c \alpha-d \beta}{\delta \mathfrak{f}}\right)\right)|N(c z+d)|^{-2-2 s}
\end{aligned}
$$

where $(c, d)$ runs over all non- $E_{f}^{+}$-associated pairs $(\neq(0,0))$ of integers. If $\operatorname{Re}(s)>0$, then the series is absolutely convergent and $G(z, s)$ is a "real analytic" function with respect to $z$. Dividing the set $\left\{(c, d)_{\uparrow}^{+}\right\}$into $\left\{(0, d)_{\mathrm{f}}^{+}\right\}$and $\left\{(c, d)_{\mathrm{f}}^{+} ; c \neq 0\right\}$, we get

$$
\begin{aligned}
G(z ; s)= & \sum_{(d)_{f}^{+}} \exp \left(2 \pi i S\left(\frac{d \beta}{\delta \mathfrak{f}}\right)\right)|N(d)|^{-2-2 s} \\
& +\sum_{(c)_{+}^{+}} \exp \left(2 \pi i S\left(\frac{c \alpha}{\delta \mathfrak{f}}\right)\right) \sum_{d \in \vartheta} \exp \left(2 \pi i S\left(-\frac{d \beta}{\delta \tilde{f}}\right)\right)|N(c z+d)|^{-2-2 s}
\end{aligned}
$$

where $\sum_{(c)_{+}^{+}}$means the summation over all non- $E_{\uparrow}^{+}$-associated integers $\neq 0$. We note the following identity:

$$
\begin{aligned}
\mid N(c z & +d)\left.\right|^{-2-2 s}=\frac{1}{N(c)\left(z_{1}-\bar{z}_{1}\right)\left(z_{2}-\bar{z}_{2}\right)}\left\{\frac{1}{\left(c_{1} \bar{z}_{1}+d_{1}\right)}-\frac{1}{\left(c_{1} z_{1}+d_{1}\right)}\right\} \\
& \times\left\{\frac{1}{\left(c_{2} \bar{z}_{2}+d_{2}\right)}-\frac{1}{\left(c_{2} z_{2}+d_{2}\right)}\right\} \frac{1}{|N(c z+d)|^{2 s}} \\
= & \frac{1}{\left(z_{1}-\bar{z}_{1}\right)\left(z_{2}-\bar{z}_{2}\right)} \frac{1}{N(c)}\left\{\frac{1}{\left(c_{1} z_{1}+d_{1}\right)\left(c_{2} z_{2}+d_{2}\right)|N(c z+d)|^{2 s}}\right. \\
& -\frac{1}{\left(c_{1} \bar{z}_{1}+d_{1}\right)\left(c_{2} z_{2}+d_{2}\right)|N(c z+d)|^{2 s}} \\
& -\frac{1}{\left(c_{1} z_{1}+d_{1}\right)\left(c_{2} \bar{z}_{2}+d_{2}\right)|N(c z+d)|^{2 s}} \\
& \left.+\frac{1}{\left(c_{1} \bar{z}_{1}+d_{1}\right)\left(c_{2} \bar{z}_{2}+d_{2}\right)|N(c z+d)|^{2 s}}\right\}
\end{aligned}
$$

This gives

$$
\begin{aligned}
G(z ; s)= & \sum_{(d)_{\dagger}^{+}} \exp \left(2 \pi i S\left(\frac{d \beta}{\delta \mathfrak{f}}\right)\right)|N(d)|^{-2-2 s}+\frac{1}{\left(z_{1}-\bar{z}_{1}\right)\left(z_{2}-\bar{z}_{2}\right)} \\
& \times\left\{F\left(z_{1}, z_{2} ; s\right)-F\left(\bar{z}_{1}, z_{2} ; s\right)-F\left(z_{1}, \bar{z}_{2} ; s\right)+F\left(\bar{z}_{1}, \bar{z}_{2} ; s\right)\right\}
\end{aligned}
$$


with

$$
\begin{aligned}
& F\left(w_{1}, w_{2} ; s\right)=F(w, \alpha, \beta ; \mathfrak{\uparrow} ; s) \\
= & \sum_{(c)+} \exp \left(2 \pi i S\left(\frac{c \alpha}{\delta \mathfrak{f}}\right)\right) N(c)^{-1} \sum_{d \in \vartheta} \exp \left(2 \pi i S\left(-\frac{d \beta}{\delta \tilde{f}}\right)\right) \frac{1}{N(c w+d)|N(c w+d)|^{2 s}}
\end{aligned}
$$

where $w_{1}, w_{2} \in \boldsymbol{C}-\boldsymbol{R}$. In the sequel we shall exclusively consider $F(w ; s)$ when $w=z \in H^{2}$ as a typical case.

Theorem 1. $F(z ; \alpha, \beta ; f ; s)$ is meromorphically continued in the whole $s$-plane and regular at $s=0$. Furthermore $F(z ; \alpha, \beta ; \mathfrak{f})=F(z ; \alpha, \beta ; \mathfrak{f} ; 0)$ is an analytic function with respect to $z \in H^{2}$;

$$
\begin{aligned}
F(z ; \alpha, \beta ; \mathfrak{f})=(2 \pi i)^{2} D^{-1 / 2} \sum_{\nu / \delta \mathfrak{f}>0} a_{\nu}(\alpha, \beta ; \mathfrak{f}) \exp \left(2 \pi i S\left(\frac{\nu}{\delta \mathfrak{f}} z\right)\right) \quad \text { if } \beta \neq 0(\mathfrak{f}), \\
=(\pi i)^{2} D^{-1 / 2} \sum_{(c)_{\mathfrak{f}}^{+}} \exp \left(2 \pi i S\left(\frac{c \alpha}{\delta \mathfrak{f}}\right)\right)|N(c)|^{-1} \\
\quad+(2 \pi i)^{2} D^{-1 / 2} \sum_{\nu / \delta \mathfrak{f}>0} a_{\nu}(\alpha, \beta ; \mathfrak{f}) \exp \left(2 \pi i S\left(\frac{\nu}{\delta \mathfrak{f}} z\right)\right) \quad \text { if } \beta \equiv 0(\mathfrak{f}) .
\end{aligned}
$$

Here $\sum_{\nu / \delta \mathrm{i}>0}$ means the summation over all integers $\nu \neq 0$ such that $\nu / \delta 千$ is totally positive, and the coeffcient $a_{\nu}(\alpha, \beta ; \mathfrak{f})$ is defined by:

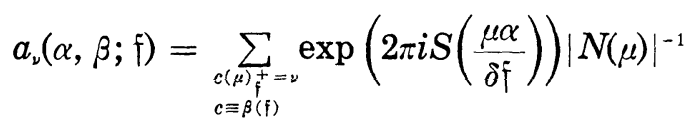

where $\mu$ runs over non- $E_{\mathrm{f}}^{+}$-associated divisors of $\nu$ under the condition $\nu / \mu$ $\equiv \beta(\tilde{f})$.

Proof. The statement follows from the routine argument for the Fourier expansion of Hecke's Eisenstein series ([3], [4]). We have

$$
\begin{aligned}
& \left.\sum_{d \equiv r(f)} \frac{1}{N(c z+d)|N(c z+d)|^{2 s}}\right|_{s=0} \\
& =(2 \pi i)^{2} f^{-1} D^{-1 / 2} \operatorname{sgn} N(c) \sum_{\nu c / \delta \uparrow>0} \exp \left(2 \pi i S\left(\frac{\nu \gamma}{\delta \digamma}\right)\right) \exp \left(2 \pi i S\left(\frac{\nu c}{\delta 千} z\right)\right) \\
& +\left.f^{-1} D^{-1 / 2} \int_{-\infty}^{\infty} \int_{-\infty}^{\infty} \frac{d u_{1} d u_{2}}{\left(c_{1} z_{1}+u_{1}\right)\left(c_{2} z_{2}+u_{2}\right)\left|\left(c_{1} z_{1}+u_{1}\right)\left(c_{2} z_{2}+u_{2}\right)\right|^{2 s}}\right|_{s=0} .
\end{aligned}
$$

Therefore if $\beta \not \equiv 0(\mathfrak{f})$, 


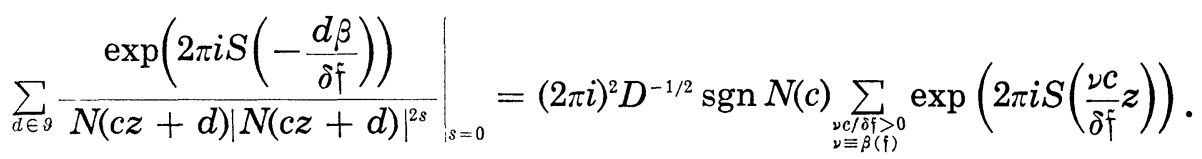

On the otherhand, in case of $\beta \equiv 0(\mathfrak{f})$, the constant term of the Fourier expansion of $F\left(z_{1}, z_{2} ; s\right)$ is given by

$$
\sum_{\langle c)_{+}^{+}} \exp \left(2 \pi i S\left(\frac{e \alpha}{\delta f}\right)\right) N(c)^{-1} D^{-1 / 2} \int_{-\infty}^{\infty} \int_{-\infty}^{\infty} \frac{d u_{1} d u_{2}}{\left(c_{1} z_{1}+u_{1}\right)\left(c_{2} z_{2}+u_{2}\right)\left|\left(c_{1} z_{1}+u_{1}\right)\left(c_{2} z_{2}+u_{2}\right)\right|^{2 s}}
$$

which reduced to

$$
(\pi i)^{2} D^{-1 / 2} \sum_{(c)_{+}^{+}} \exp \left(2 \pi i S\left(\frac{c \alpha}{\delta f}\right)\right)|N(c)|^{-1}
$$

at $s=0$, and the other terms are the similar ones to the case of $\beta \not \equiv(\mathfrak{f})$.

§2. $\Phi(z ; \alpha, \beta ; \mathfrak{\uparrow})$

We introduce an analytic function on $z \in H^{2}$, defined by

$$
\Phi(z ; \alpha, \beta ; \mathfrak{f})=q(2, \beta \bmod \mathfrak{\dagger}) N(z)+F(z ; \alpha, \beta ; \mathfrak{f})
$$

where $q(2, \beta \bmod \mathfrak{f})=\sum_{(c)_{\dagger}^{+}} \exp (2 \pi i S(c \beta / \delta \tilde{f}))|N(c)|^{-2}$. It is easy to see that

$$
\begin{aligned}
& \Phi(z+\nu ; \alpha, \beta ; \mathfrak{f}) \\
& \quad=\Phi(z ; \alpha+\beta \nu, \beta ; \mathfrak{f})+q(2, \beta \bmod \mathfrak{f})\left(\nu_{1} z_{2}+\nu_{2} z_{1}+N(\nu)\right) \quad(\nu \in \vartheta)
\end{aligned}
$$

and that

$$
\Phi(\eta z ; \alpha, \beta ; \mathfrak{f})=\Phi(z ; \alpha, \beta ; \mathfrak{f}) \quad\left(\eta \in E_{\uparrow}^{+}\right) .
$$

We shall see a simple relation between $\Phi(z ; \alpha, \beta ; f)$ and Eisenstein series on $H^{2}$. Let us consider kind of Eisenstein series; for $s \in C$ and $z \in H^{2}$,

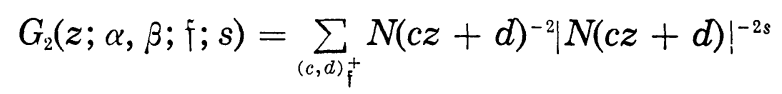

where $(c, d)$ runs over all non- $E_{\mathrm{f}}^{+}$-associated pairs $\neq(0,0)$ of integers subject to $c \equiv \alpha(\mathfrak{f})$ and $d \equiv \beta(\mathfrak{f})$. It is known that $G_{2}(z ; \alpha, \beta ; \mathfrak{f} ; s)$ is meromorphically continued to the whole $s$-plane and regular at $s=0$ and that $G_{2}(z ; \alpha, \beta ; \mathfrak{f})=G_{2}(z ; \alpha, \beta ; \mathfrak{f} 0)$ is an analytic function on $z \in H^{2}$ (see [5]). Furthermore, if $a, b, c, d \in \vartheta, a d-b c \in E^{+}$(the group of all totally positive units of $K$ ), then 


$$
G_{2}\left(\frac{a z+b}{c z+d} ; \alpha, \beta ; \mathfrak{f}\right) N(c z+d)^{-2}=G_{2}(z ; a \alpha+c \beta, b \alpha+d \beta ; \mathfrak{f}) .
$$

We put

$$
G_{2}^{*}(z ; \alpha, \beta ; \mathfrak{f})=\sum_{\gamma, \tilde{o}^{\prime} \bmod \mathfrak{f}} \exp \left(2 \pi i S\left(\frac{\alpha \gamma-\beta \delta^{\prime}}{\delta \mathfrak{f}}\right)\right) G_{2}\left(z ; \gamma, \delta^{\prime} ; \mathfrak{f}\right)
$$

where $\gamma$ and $\delta^{\prime}$ each run through a complete representative set of residue classes $\bmod \mathfrak{f}$. Then by direct calculations of their Fourier expansions we get

$$
\frac{\partial^{2}}{\partial z_{1} \partial z_{2}} \Phi(z ; \alpha, \beta ; \mathfrak{f})=G_{2}^{*}(z ; \alpha, \beta ; \mathfrak{f})
$$

Now we investigate the behavior of $\Phi(z ; \alpha, \beta ; \hat{f})$ under the substitution $z \rightarrow-z^{-1}=\left(-z_{1}^{-1},-z_{2}^{-1}\right)$. Let $v$ denote one of the characters $v_{i}(i=0,1,2,3)$ defined by $v_{0}(\nu)=1, v_{1}(\nu)=\operatorname{sgn} N(\nu), v_{2}(\nu)=\operatorname{sgn} \nu_{1}$ and $v_{3}(\nu)=\operatorname{sgn} \nu_{2}$ for $\nu \in \vartheta$ $(\neq 0)$, respectively. We consider an auxiliary function defined by

$$
\begin{aligned}
H(t ; v) & =H(t ; \alpha, \beta ; \mathfrak{f} ; v) \\
& =\sum_{\nu \neq 0} a_{\nu}(\alpha, \beta ; \mathfrak{f}) v(\nu) \exp \left(-t_{1}\left|\nu_{1}\right|-t_{2}\left|\nu_{2}\right|\right)
\end{aligned}
$$

where $t=\left(t_{1}, t_{2}\right) \in C^{2}\left(\operatorname{Re}\left(t_{1}\right)>0, \operatorname{Re}\left(t_{2}\right)>0\right), a_{\nu}(\alpha, \beta ; f)$ is defined by (1.6) and $\nu$ runs over all integers $\neq 0$ of $\vartheta$. It is easy to see that

with

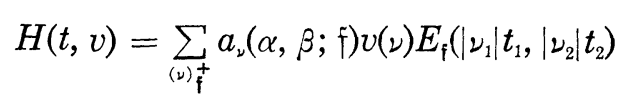

$$
E_{\mathrm{f}}\left(t_{1}, t_{2}\right)=\sum_{n=-\infty}^{\infty} \exp \left(-t_{1}\left|\eta_{\mathrm{f}}\right|^{n}-t_{2}\left|\eta_{\mathrm{f}}\right|^{-n}\right)
$$

where $\eta_{\mathrm{f}}$ is the generator of $E_{\mathrm{f}}^{+}$such that $\eta_{\mathrm{f}}>1$. By virtue of [3], $E_{\mathrm{f}}\left(t_{1}, t_{2}\right)$ is expressed in the following form:

$$
E_{\mathrm{f}}\left(t_{1}, t_{2}\right)=\left(2 \pi i \log \eta_{\mathrm{f}}\right)^{-1} \sum_{n=-\infty}^{\infty} \int_{2-i \infty}^{2+i \infty} \frac{\Gamma(s+i n b) \Gamma(s-i n b)}{t_{1}^{s+i n b} t_{2}^{s-i n b}} d s \quad\left(b=\frac{\pi}{\log \eta_{\mathrm{f}}}\right) .
$$

This gives

$$
H(t, v)=\left(2 \pi i \log \eta_{i}\right)^{-1} \sum_{n=-\infty}^{\infty} A_{n}(t, v)
$$

with 


$$
\begin{aligned}
A_{n}(t, v) & =A_{n}(t ; \alpha, \beta ; \mathfrak{f} ; v) \\
& =\int_{2-i \infty}^{2+i \infty} \frac{\Gamma(s+i n b) \Gamma(s-i n b)}{t_{1}^{s+i n b} t_{2}^{s-i n b}} Z_{n}(s, v) d s
\end{aligned}
$$

where

$$
\begin{aligned}
Z_{n}(s, v) & =Z_{n}(s ; \alpha, \beta ; \mathfrak{f} ; v) \\
& =\sum_{(\nu)_{\mathfrak{f}}^{+}} \frac{a_{\nu}(\alpha, \beta ; \mathfrak{f}) v(\nu)}{\left|\nu_{1}\right|^{s+i n b}\left|\nu_{2}\right|^{s-i n b}} .
\end{aligned}
$$

We put

$$
\lambda_{n}(\nu)=\left|\frac{\nu_{2}}{\nu_{1}}\right|^{i n b} \quad \text { for } \nu \in \vartheta(\neq 0)
$$

Then $\left(\lambda_{n} v\right)(\eta)=1$ for all $\eta \in E_{\mathrm{f}}^{+}$; this means $\left(\lambda_{n} v\right)$ is a Grössen-character defined modulo $f$ (in Hecke's original sense). We have

$$
\begin{aligned}
Z_{n}(s, v) & =Z_{n}(s ; \alpha, \beta ; \mathfrak{f} ; v) \\
& =\sum_{\substack{(c)_{\dagger}^{+} \\
c \equiv \beta(\mathfrak{f})}}\left(\lambda_{n} v\right)(c)|N(c)|^{-s} \sum_{\substack{(\mu)+\\
\uparrow}}\left(\lambda_{n} v\right)(\mu) \exp \left(2 \pi i S\left(\frac{\alpha \mu}{\delta \tilde{f}}\right)\right)|N(\mu)|^{-s-1} .
\end{aligned}
$$

In view of (2.9), we set

$$
P\left(s, \alpha \bmod \mathfrak{f}, \lambda_{n} v\right)=(D f)^{s / 2} \pi^{-s} \gamma\left(s ; \lambda_{n} v\right) \sum_{\substack{(\mu) \uparrow_{\uparrow}^{+} \\ \mu \equiv \alpha(\mathfrak{f})}}\left(\lambda_{n} v\right)(\mu)|N(\mu)|^{-s},
$$

$$
Q\left(s, \alpha \bmod \mathfrak{f}, \lambda_{n} v\right)
$$

$$
=\left(\lambda_{n} v\right)(\delta \mathfrak{f}) f^{-1 / 2}(D f)^{s / 2} \pi^{-s} \gamma\left(s ; \lambda_{n} v\right) \sum_{(\mu)_{f}^{+}}\left(\lambda_{n} v\right)(\mu) \exp \left(2 \pi i S\left(\frac{\alpha \mu}{\delta \mathfrak{f}}\right)\right)|N(\mu)|^{-s}
$$

where

$$
\begin{aligned}
\gamma\left(s ; \lambda_{n} v\right) & =\Gamma\left(\frac{s+i n b}{2}\right) \Gamma\left(\frac{s-i n b}{2}\right) & & \text { if } v=v_{0}, \\
& =\Gamma\left(\frac{s+1+i n b}{2}\right) \Gamma\left(\frac{s+1-i n b}{2}\right) & & \text { if } v=v_{1}, \\
& =\Gamma\left(\frac{s+i n b}{2}\right) \Gamma\left(\frac{s+1-i n b}{2}\right) & & \text { if } v=v_{2}, \\
& =\Gamma\left(\frac{s+1+i n b}{2}\right) \Gamma\left(\frac{s-i n b}{2}\right) & & \text { if } v=v_{3} .
\end{aligned}
$$

We shall prove the following lemma in the next section. 
LEMma. If $\alpha \neq 0(\mathfrak{f})$, then $P\left(s, \alpha \bmod \mathfrak{f}, \lambda_{n} v\right)$ is analytically continued in the whole s-plane except a pole at $s=1$ in case of $\lambda_{n} v=v_{0}$; and

$$
\operatorname{Res}_{s=1} P\left(s, \alpha \bmod \mathfrak{f}, v_{0}\right)=4 f^{-1 / 2} \log \eta_{\mathfrak{f}} .
$$

$P\left(s, 0 \bmod \mathfrak{f}, \lambda_{n} v\right)$ is analytically continued in the whole s-plane except poles at $s=0,1$ in case of $\lambda_{n} v=v_{0}$; and

$$
\begin{aligned}
& \operatorname{Res}_{s=1} P\left(s, 0 \bmod \mathfrak{f}, v_{0}\right)=4 f^{-1 / 2} \log \eta_{\mathfrak{f}}, \\
& \operatorname{Res}_{s=0} P\left(s, 0 \bmod \mathfrak{f}, v_{0}\right)=-4 \log \eta_{\mathfrak{f}} .
\end{aligned}
$$

Furthermore, the following functional equation holds:

$$
P\left(s, \alpha \bmod \mathfrak{f}, \lambda_{n} v\right)=i^{-k(v)} Q\left(1-s, \alpha \bmod \mathfrak{f}, \bar{\lambda}_{n} v\right)
$$

where $k(v)=0,2,1$ for $v=v_{0}, v_{1}, v_{2}$ (or $\left.v_{3}\right)$, respectively.

Making use of an expression of $Z_{n}(s, v)$ by $P(s)$ and $Q(s)$ and the functional equation of $P(s)$ and $Q(s)$, we get a transformation formula for $A_{n}(t, v)$.

[1] the case of $v=v_{0}$. Since

$$
\Gamma\left(\frac{s}{2}\right) \Gamma\left(\frac{s}{2}+\frac{1}{2}\right)=2^{1-s} \pi^{1 / 2} \Gamma(s),
$$

it follows from (2.7), (2.9), (2.10) and (2.11) that

$$
\begin{aligned}
A_{n}(t, v) & =4^{-1} D^{-1 / 2} \int_{2-i \infty}^{2+i \infty} \frac{P\left(s, \beta \bmod \mathfrak{f}, \lambda_{n}\right) Q\left(s+1, \alpha \bmod \mathfrak{f}, \lambda_{n}\right)}{(2 \pi)^{-2 s}(D f)^{s} \lambda_{n}(\delta \mathfrak{f}) t_{1}^{s+i n b} t_{2}^{s-i n b}} d s \\
& =4^{-1} D^{-1 / 2} \int_{2-i \infty}^{2+i \infty} \frac{P\left(s, \beta \bmod \mathfrak{f}, \lambda_{n}\right) Q\left(s+1, \alpha \bmod \mathfrak{f}, \lambda_{n}\right)}{t_{1}^{\prime s+i n b} t_{2}^{\prime s-i n b}} d s
\end{aligned}
$$

where $t_{1}^{\prime}=\left(\left|\delta_{1} \mathfrak{F}_{1}\right| / 2 \pi\right) t_{1}$ and $t_{2}^{\prime}=\left(\left|\delta_{2} \mathfrak{f}_{2}\right|\right) t_{2}$. We change the integral path from $\operatorname{Re}(s)=2$ to $\operatorname{Re}(s)=-2$, and substitute $s$ by $-s$. Then it follows from the functional equation in Lemma that

$$
\begin{aligned}
4 D^{1 / 2} A_{n}\left(t ; \alpha, \beta ; \mathfrak{f} ; v_{0}\right)= & \int_{-2-i \infty}^{-2+i \infty} \frac{P\left(s, \beta \bmod \mathfrak{f}, \lambda_{n}\right) Q\left(s+1, \alpha \bmod \mathfrak{f}, \lambda_{n}\right)}{t_{1}^{\prime s+i n b} t_{2}^{\prime s-i n b}} d s \\
& +2 \pi i(\operatorname{sum} \text { of residues of the integrand in } \\
& -2<\operatorname{Re}(s)<2) \\
= & \int_{2-i \infty}^{2+i \infty} \frac{Q\left(s+1, \beta \bmod \mathfrak{f}, \bar{\lambda}_{n}\right) P\left(s, \alpha \bmod \mathfrak{f}, \bar{\lambda}_{n}\right)}{t_{1}^{\prime-s+i n b} t_{2}^{\prime-s-i n b}} d s \\
& +2 \pi i \text { (sum of residues). }
\end{aligned}
$$


If $n \neq 0$, then the integrand has no poles in $-2<\operatorname{Re}(s)<2$, so we get

$$
A_{n}\left(t_{1}, t_{2} ; \alpha, \beta ; \mathfrak{f} ; v_{0}\right)=A_{-n}\left(\frac{4 \pi^{2}}{\left|\delta_{1} \tilde{f}_{1}\right|^{2} t_{1}}, \frac{4 \pi^{2}}{\left|\delta_{2} \mathfrak{f}_{2}\right|^{2} t_{2}} ; \beta, \alpha ; \mathfrak{f} ; v_{0}\right) \text {. }
$$

If $n=0$, then the residues of the integrand in $-2<\operatorname{Re}(s)<2$ are as follows:

at $s=1, \quad 4 f^{-1 / 2} \log \eta_{\mathfrak{f}} Q\left(2, \alpha \bmod \mathfrak{f}, v_{0}\right)\left(t_{1}^{\prime} t_{2}^{\prime}\right)^{-1}$

$$
=4 D \pi^{-2} \log \eta_{\mathrm{i}} q(2, \alpha \bmod f)\left(t_{1}^{\prime} t_{2}^{\prime}\right)^{-1} \text {; }
$$

at $s=0, \quad-\Delta(\beta ; \mathfrak{f}) 4 \log \eta_{\mathrm{f}} Q\left(1, \alpha \bmod \mathfrak{f}, v_{0}\right)+\Delta(\alpha, \mathfrak{f}) 4 \log \eta_{\mathrm{f}} Q\left(1, \beta \bmod \mathfrak{f}, v_{0}\right)$

$$
=-(\beta, f) 4 D^{1 / 2} \log \eta_{\mathrm{i}} q(1, \alpha \bmod f)+\Delta(\alpha, f) 4 D^{1 / 2} \log \eta_{\mathrm{f}} q(1, \beta \bmod f) \text {; }
$$

at $s=-1, \quad-4 \pi^{-2} D \log \eta_{\uparrow} q(2, \beta \bmod f) t_{1}^{\prime} t_{2}^{\prime}$

where

$$
q(s, \alpha \bmod f)=\sum_{(c)^{+}} \exp \left(2 \pi i S\left(\frac{c \alpha}{\delta \digamma}\right)\right)|N(c)|^{-s}
$$

and

$$
\begin{array}{rlrl}
\Delta(\alpha ; \mathfrak{f})=1 & & \text { if } \alpha \equiv 0(\mathfrak{f}), \\
& =0 & & \text { if } \alpha \neq 0(\mathfrak{f}) .
\end{array}
$$

So we get

$$
\begin{aligned}
& A_{0}\left(t_{1}, t_{2} ; \alpha, \beta ; \mathfrak{f} ; v_{0}\right)=A_{0}\left(\frac{4 \pi^{2}}{\left|\delta_{1} \mathfrak{f}_{1}\right|^{2} t_{1}} \frac{4 \pi^{2}}{\left|\delta_{2} \mathfrak{f}_{2}\right|^{2} t_{2}} ; \beta, \alpha ; \mathfrak{f} ; v_{0}\right) \\
& \quad+2 \pi i \log \eta_{\mathfrak{f}}\left[\frac{D^{1 / 2}}{\pi^{2}}\left\{q(2, \alpha \bmod \mathfrak{f}) \frac{1}{t_{1}^{\prime} t_{2}^{\prime}}-q(2, \beta \bmod \mathfrak{f}) t_{1}^{\prime} t_{2}^{\prime}\right\}\right. \\
& \quad-\Delta(\beta ; \mathfrak{f}) q(1, \alpha \bmod \mathfrak{f})+\Delta(\alpha ; \mathfrak{f}) q(1, \beta \bmod \mathfrak{f})] .
\end{aligned}
$$

[2] the case of $v=v_{i}$. We have

$$
A_{n}\left(t_{1}, t_{2} ; v_{1}\right)=v_{1}(\delta \mathfrak{f}) D^{-1 / 2} \int_{2-i \infty}^{2+i \infty} \frac{P\left(s, \beta \bmod \mathfrak{F}, \lambda_{n} v_{1}\right) Q\left(s+1, \alpha \bmod \mathfrak{f}, \lambda_{n} v_{1}\right)}{\left(s^{2}+n^{2} b^{2}\right) t_{1}^{\prime s+i n b} t_{2}^{\prime s-i n b}} d s .
$$

There exist infinitely many poles of the integrand in $-2<\operatorname{Re}(s)<2$. If $n=0$, the residue at $s=0$ is

$$
\begin{aligned}
& -P\left(0, \beta \bmod \mathfrak{f}, v_{1}\right) Q\left(1, \alpha \bmod \mathfrak{f}, v_{1}\right) \log \left(t_{1}^{\prime} t_{2}^{\prime}\right) \\
= & D \pi^{-2} q\left(1, \beta \bmod \mathfrak{f}, v_{1}\right) q\left(1, \alpha \bmod \mathfrak{f}, v_{1}\right) \log \left(t_{1}^{\prime} t_{2}^{\prime}\right) ;
\end{aligned}
$$

if $n \neq 0$, the sum of the residues at $s=i n b$ and $s=-i n b$ is 


$$
\begin{aligned}
\frac{t_{1}^{\prime-2 i n b}}{2 i n b} P\left(i n b, \beta \bmod \mathfrak{f}, \lambda_{n} v_{1}\right) Q\left(1+i n b, \alpha \bmod \mathfrak{f}, \lambda_{n} v_{1}\right) \\
\quad-\frac{t_{2}^{\prime 2 i n b}}{2 i n b} P\left(-i n b, \beta \bmod \mathfrak{f}, \lambda_{n} v_{1}\right) Q\left(1-i n b, \alpha \bmod \mathfrak{f}, \lambda_{n} v_{1}\right) \\
=-\frac{D}{2 \pi \sin (\pi i n b)} t_{1}^{\prime-2 i n b} q\left(1-i n b, \beta \bmod \mathfrak{f}, \bar{\lambda}_{n} v_{1}\right) q\left(1+i n b, \bmod \tilde{\mathfrak{f}}, \lambda_{n} v_{1}\right) \\
\quad+\frac{D}{2 \pi \sin (\pi i n b)} t_{2}^{\prime 2 i n b} q\left(1+i n b, \beta \bmod \mathfrak{f}, \bar{\lambda}_{n} v_{1}\right) q\left(1-i n b, \alpha \bmod \mathfrak{f}, \lambda_{n} v_{1}\right)
\end{aligned}
$$

where

$$
q\left(s, \alpha \bmod \mathfrak{f}, \lambda_{n} v\right)=\sum_{(c)_{\tilde{f}}^{+}} \exp \left(2 \pi i S\left(\frac{\alpha v}{\delta \tilde{f}}\right)\right)\left(\lambda_{n} v\right)(c)|N(c)|^{-s} .
$$

[3] the case of $v=v_{2}\left(\right.$ or $\left.v_{3}\right)$. We have

$$
A_{n}\left(t_{1}, t_{2} ; v\right)=v(\delta \mathfrak{f}) D^{-1 / 2} \int_{2-i \infty}^{2+i \infty} \frac{P\left(s, \beta \bmod \mathfrak{f}, \lambda_{n} v\right) Q\left(s+1, \alpha \bmod \mathfrak{f}, \lambda_{n} v\right)}{2(s \mp i n b) t_{1}^{\prime s+i n b} t_{2}^{\prime s-i n b}} d s .
$$

There exist infinitely many poles of the integrand in $-2<\operatorname{Re}(s)<2$. The residue at $s=\operatorname{inb}(s=-i n b)$ is

$$
\begin{aligned}
& \frac{1}{2 i_{1}^{\prime 2 i n b}} P\left(i n b, \beta \bmod \mathfrak{f}, \lambda_{n} v_{2}\right) Q\left(1+i n b, \alpha \bmod \mathfrak{f}, \lambda_{n} v_{2}\right) \\
&= \frac{-i D}{2 \pi \cos (\pi i n b)} t_{1}^{\prime-2 i n b} q\left(1-i n b, \beta \bmod \mathfrak{f}, \bar{\lambda}_{n} v_{2}\right) q\left(1+i n b, \bmod \tilde{\mathfrak{f}}, \lambda_{n} v_{2}\right) \\
&\left(\begin{array}{l}
\frac{1}{2 t_{2}^{\prime-2 i n b}} P\left(-i n b, \beta \bmod \mathfrak{f}, \lambda_{n} v_{3}\right) Q\left(1-i n b, \alpha \bmod \mathfrak{f}, \lambda_{n} v_{3}\right) \\
=\frac{-i D}{2 \pi \cos (\pi i n b)} t_{2}^{\prime 2 i n b} q\left(1+i n b, \beta \bmod \mathfrak{f}, \bar{\lambda}_{n} v_{3}\right) q\left(1-i n b, \alpha \bmod \mathfrak{f}, \lambda_{n} v_{3}\right)
\end{array}\right) .
\end{aligned}
$$

If we put

$$
H^{\prime}\left(t_{1}, t_{2} ; \alpha, \beta ; \mathfrak{f} ; v\right)=H\left(\frac{2 \pi t_{1}}{\left|\delta_{1} \tilde{f}_{1}\right|}, \frac{2 \pi t_{2}}{\left|\delta_{2} f_{2}\right|} ; \alpha, \beta ; \mathfrak{\mathfrak { f }} ; v\right)
$$

then by (2.6), (2.13) and (2.14) we have

$$
\begin{aligned}
& H^{\prime}\left(t_{1}, t_{2} ; \alpha, \beta ; \mathfrak{\uparrow} ; v_{0}\right)=H^{\prime}\left(t_{1}^{-1}, t_{2}^{-} ; \beta, \alpha ; \mathfrak{\uparrow} ; v_{0}\right) \\
& +\frac{D^{1 / 2}}{\pi^{2}}\left\{q(2, \alpha \bmod \tilde{i}) \frac{1}{t_{1} t_{2}}-q(2, \beta \bmod \tilde{i}) t_{1} t_{2}\right\} \\
& -\Delta(\beta ; \mathfrak{f}) q(1, \alpha \bmod \tilde{i})+\Delta(\alpha ; \mathfrak{i}) q(1, \beta \bmod \tilde{T}) \text {. }
\end{aligned}
$$


Similarly we have

$$
\begin{aligned}
& v_{1}(\delta \mathfrak{f}) H^{\prime}\left(t^{1}, t_{2} ; \alpha, \beta ; \mathfrak{f} ; v_{1}\right)=v_{1}(\delta \mathfrak{f}) H^{\prime}\left(t_{1}^{-1}, t_{2}^{-1} ; \beta, \alpha ; \mathfrak{f} ; v_{1}\right) \\
& -\frac{D^{1 / 2}}{\pi^{2} \log \eta_{\mathfrak{f}}} q\left(1, \beta \bmod \mathfrak{f}, v_{1}\right) q\left(1, \alpha \bmod \mathfrak{f}, v_{1}\right) \log \left(t_{1} t_{2}\right) \\
& -\frac{D^{1 / 2}}{\pi^{2}} \sum_{n \neq 0}\left\{g_{1}(n ; \alpha, \beta ; \mathfrak{f}) t_{1}^{-2 i n b}+g_{2}(n ; \alpha, \beta ; \mathfrak{f}) t_{2}^{-2 i n b}\right\} \\
& v_{2}(\delta \mathfrak{f}) H^{\prime}\left(t, t_{2} ; \alpha, \beta ; \mathfrak{f} ; v_{2}\right)=v_{2}(\delta \mathfrak{f}) H^{\prime}\left(t_{1}^{-1}, t_{2}^{-1} ; \beta, \alpha ; \mathfrak{f} ; v_{2}\right) \\
& \quad-\frac{D^{1 / 2}}{\pi^{2}} \sum_{n=-\infty}^{\infty} g_{3}(n ; \alpha, \beta ; \mathfrak{f}) t_{1}^{-2 i n b},
\end{aligned}
$$

and

$$
\begin{aligned}
& v_{3}(\delta \mathfrak{f}) H^{\prime}\left(t_{1}, t_{2} ; \alpha, \beta ; \mathfrak{f} ; v_{3}\right)=v_{3}(\delta \mathfrak{f}) H^{\prime}\left(t_{1}^{-1}, t_{2}^{-1} ; \beta, \alpha ; \mathfrak{f} ; v_{3}\right) \\
& -\frac{D^{1 / 2}}{\pi^{2}} \sum_{n=-\infty}^{\infty} g_{3}(n ; \alpha, \beta ; \mathfrak{f}) t_{2}^{-2 i n b}
\end{aligned}
$$

where

$$
\begin{aligned}
& g_{1}(n ; \alpha, \beta ; \mathfrak{f})=\frac{q\left(1-i n b, \beta \bmod \mathfrak{f}, \bar{\lambda}_{n} v_{1}\right) q\left(1+i n b, \alpha \bmod \mathfrak{f}, \lambda_{n} v_{1}\right)}{2 \pi^{-1} \log \eta_{\mathfrak{f}} \sin (\pi i n b)}, \\
& g_{2}(n ; \alpha, \beta ; \mathfrak{f})=\frac{q\left(1-i n b, \beta \bmod \mathfrak{f}, \lambda_{n} v_{1}\right) q\left(1+i n b, \alpha \bmod \mathfrak{f}, \bar{\lambda}_{n} v_{1}\right)}{2 \pi^{-1} \log \eta_{\mathfrak{f}} \sin (\pi i n b)}, \\
& g_{3}(n ; \alpha, \beta ; \mathfrak{f})=i \frac{q\left(1-i n b, \beta \bmod \mathfrak{f}, \bar{\lambda}_{n} v_{2}\right) q\left(1+i n b, \alpha \bmod \mathfrak{f}, \lambda_{n} v_{2}\right)}{2 \pi \log \eta_{\mathfrak{f}} \cos (\pi \operatorname{in} b)}, \\
& g_{4}(n ; \alpha, \beta ; \mathfrak{f})=i \frac{q\left(1-i n b, \beta \bmod \mathfrak{f}, \lambda_{n} v_{3}\right) q\left(1+i n b, \alpha \bmod \mathfrak{f}, \bar{\lambda}_{n} v_{3}\right)}{2 \pi^{-1} \log \eta_{\mathfrak{f}} \cos (\pi \operatorname{in} b)} .
\end{aligned}
$$

It follows from (2.5) and (2.15) that

$$
\sum_{0 \leq j \leq 3} v_{j}(\delta \mathfrak{f}) H^{\prime}\left(t_{1}, t_{2} ; v_{j}\right)=4 \sum_{\nu / \delta \uparrow>0} a_{\nu}(\alpha, \beta ; \mathfrak{f}) \exp \left(-2 \pi\left(\frac{t_{1} \nu_{1}}{\delta_{1} \mathfrak{f}_{1}}+\frac{t_{2} \nu_{2}}{\delta_{2} \mathfrak{f}_{2}}\right)\right)
$$

and so

$$
\begin{aligned}
& E\left(z_{1}, z_{2} ; \alpha, \beta ; \uparrow\right)=(\pi i)^{2} D^{-1 / 2} \sum_{0 \leqq j \leqq 3} v_{j}(\delta \uparrow) H^{\prime}\left(\frac{z_{1}}{i}, \frac{z_{2}}{i} ; v_{j}\right) \\
& +\Delta(\beta ; \mathfrak{f})(\pi i)^{2} D^{-1 / 2} q(1, \alpha \bmod \mathfrak{f}) \text {. }
\end{aligned}
$$

By (2.1), (2.16), (2.17), (2.18), (2.19) and (2.20) we obtain

\section{THEOREM 2. We have}




$$
\begin{aligned}
\Phi(- & \left.z_{1}^{-1},-z_{2}^{-1} ; \beta, \alpha ; \mathfrak{f}\right) \\
= & \Phi\left(z_{1} z_{2} ; \alpha, \beta ; \mathfrak{f}\right)-\frac{1}{\log \eta_{\mathfrak{f}}} q(1, \beta \bmod \mathfrak{f}) q(1, \alpha \bmod \mathfrak{f})\left\{\log z_{1}+\log z_{2}-i \pi\right\} \\
& \quad-\sum_{n=-\infty}^{\infty}\left\{g(n ; \alpha, \beta ; \mathfrak{f}) e^{-\pi n b} z_{1}^{-2 i n b}+h(n ; \alpha, \beta ; \tilde{\mathfrak{f}}) e^{-\pi n b} z_{2}^{-2 i n b}\right\}
\end{aligned}
$$

where

$$
\begin{aligned}
g(n ; \alpha, \beta ; \mathfrak{f}) & =g_{1}(n ; \alpha, \beta ; \mathfrak{f})+g_{3}(n ; \alpha, \beta ; \mathfrak{f}) & & \text { if } n \neq 0, \\
& =g_{3}(0 ; \alpha, \beta ; \mathfrak{\dagger}) & & \text { if } n=0, \\
h(n ; \alpha, \beta ; \mathfrak{\dagger}) & =g_{2}(n ; \alpha, \beta ; \mathfrak{f})+g_{4}(n ; \alpha, \beta ; \mathfrak{f}) & & \text { if } n \neq 0, \\
& =g_{4}(0 ; \alpha, \beta ; \mathfrak{\dagger}) & & \text { if } n=0,
\end{aligned}
$$

and $z^{s}=e^{s \log z}(\operatorname{Im}(z)>0), 0<\operatorname{Im}(\log z)<\pi$.

\section{§ 3. Proof of Lemma}

Let $E$ be the group of all units of $K, E^{+}$the group of all totally positive units of $K$ and $E_{\mathrm{f}}$ the group of all units congruent 1 modulo $\tilde{\uparrow}$. Let $\eta$ denote the generator of $E^{+}(\eta>1)$. Put

$$
e(\mathfrak{f})=\left[E_{\mathfrak{i}}: E_{\mathfrak{f}}^{+}\right], \quad e^{\prime}(\mathfrak{f})=\left[E: E_{\mathfrak{i}}\right] \text { and } e(1)=\left[E: E^{+}\right],
$$

then we have

$$
\frac{\log \eta_{\mathfrak{f}}}{e(\mathfrak{T})}=\frac{e^{\prime}(\tilde{\mathfrak{f}})}{e(1)} \log \eta
$$

Let us recall the properties of Hecke's $L$-function for $K$. For $\lambda_{n} v$, consider the characters $\chi$ defined modulo $f$ such that $\lambda_{n} v_{x}$ is an ideal character modulo $\mathfrak{f}$. The number of such characters $\chi$ is equal to $(f-1) / e^{\prime}(\mathfrak{f})$. If one of such characters is $1_{f}$ (the identity character modulo f), then $\lambda_{n} v$ itself must be an ideal character. For $\chi \neq 1_{\mathfrak{f}}, \lambda_{n} v \chi$ is primitive because $\mathfrak{f}$ is prime. For ideal characters $\lambda_{n} v \chi\left(\chi \neq 1_{\mathfrak{f}}\right)$ and $\lambda_{n} v$, we set

$$
\begin{aligned}
& \Lambda\left(s, \lambda_{n} v \chi\right)=\pi^{-s}(D f)^{s / 2} \gamma\left(s ; \lambda_{n} v\right) \sum_{\substack{(c) \\
(c, f)=1}}\left(\lambda_{n} v \chi\right)(c)|N(c)|^{-s}, \\
& \Lambda\left(s, \lambda_{n} v\right)=\pi^{-s} D^{s / 2} \gamma\left(s ; \lambda_{n} v\right) \sum_{(c)}\left(\lambda_{n} v\right)(c)|N(c)|^{-s}
\end{aligned}
$$

where $\sum_{(c)}$ means the summation over all ideals of $\vartheta$; they are analytically continued in the whole $s$-plane except that $\Lambda\left(s, v_{0}\right)$ has two poles at $s=0,1$, and satisfy the following functional equations:

$$
\Lambda\left(s, \lambda_{n} v \chi\right)=i^{-k(v)} G(\chi) f^{-1 / 2}\left(\lambda_{n} v\right)\left(\delta\lceil) \Lambda\left(1-s, \bar{\chi} \bar{\lambda}_{n} v\right),\right.
$$




$$
\Lambda\left(s, \lambda_{n} v\right)=i^{-k(v)}\left(\lambda_{n} v\right)(\delta) \Lambda\left(1-s, \bar{\lambda}_{n} v\right)
$$

where $G(\chi)=\sum_{\alpha \bmod f} \chi(\alpha) e^{2 \pi i S(\alpha / \delta \mathrm{f})}$; the residues of $\Lambda\left(s, v_{0}\right)$ at $s=0$ and $s=1$ are respectively given by $-4 \log \eta / e(1)$ and $4 \log \eta / e(1)$.

We shall see that $P(s, \alpha \bmod \mathfrak{f}, v)$ and $Q(s, \alpha \bmod \mathfrak{f}, v)$ are expressed in terms of $\Lambda(s)$. Then Lemma follows from the properties of $\Lambda(s)$. If $\alpha \equiv$ $0(\tilde{\mathrm{f}})$, then by $(3.3)$

$$
\begin{aligned}
P\left(s, 0 \bmod \mathfrak{f}, \lambda_{n} v\right) & =\pi^{-s}(D f)^{s / 2} \gamma\left(s, \lambda_{n} v\right) e(\mathfrak{f}) e^{\prime}(\mathfrak{f}) \sum_{\substack{(c) \\
c \equiv 0(\mathfrak{f})}}\left(\lambda_{n} v\right)(c)|N(c)|^{-s} \\
& =e(\mathfrak{f}) e^{\prime}(\mathfrak{f}) f^{-s / 2}\left(\lambda_{n} v\right)(\mathfrak{f}) \Lambda\left(s, \lambda_{n} v\right) \\
& =i^{-k(v)} e(\mathfrak{f}) e^{\prime}(\mathfrak{f}) f^{-s / 2}\left(\lambda_{n} v\right)(\delta \mathfrak{f}) \Lambda\left(1-s, \bar{\lambda}_{n} v\right) \\
& =i^{-k(v)} Q\left(1-s, 0 \bmod \mathfrak{f}, \bar{\lambda}_{n} v\right)
\end{aligned}
$$

$P\left(s, 0 \bmod \mathfrak{f}, \lambda_{n} v\right)$ is continued analytically in the whole $s$-plane except poles at $s=0$ and $s=1$ in case of $\lambda_{n} v=v_{0}$;

$$
\begin{aligned}
\operatorname{Res}_{s=1} P\left(s, 0 \bmod \mathfrak{f}, v_{0}\right) & =e(\mathfrak{f}) e^{\prime}(\mathfrak{f}) f^{-1 / 2} 4 \log \eta / e(1) \\
& =4 f^{-1 / 2} \log \eta_{\mathfrak{f}} \quad(\text { by }(3.1)),
\end{aligned}
$$

$\operatorname{Res}_{s=0} P\left(s, 0 \bmod \mathfrak{f}, v_{0}\right)=-4 \log \eta_{\mathfrak{i}}$.

For our purpose we modify the functional equation of $\Lambda\left(s, \lambda_{n} v\right)$. If we write

$$
\begin{aligned}
& \Lambda\left(s, 1_{\mathfrak{f}} \lambda_{n} v\right)=\pi^{-s}(D f)^{s / 2} \gamma\left(s ; \lambda_{n} v\right) \sum_{\substack{(c) \\
(c, \mathfrak{f})=1}}\left(\lambda_{n} v\right)(c)|N(c)|^{-s}, \\
& \Lambda\left(s, 0_{\mathfrak{f}} \lambda_{n} v\right)=\pi^{-s}(D f)^{s / 2} \gamma\left(s ; \lambda_{n} v\right) \sum_{\substack{(c) \\
c \equiv 0(\mathfrak{f})}}\left(\lambda_{n} v\right)(c)|N(c)|^{-s},
\end{aligned}
$$

then by (3.3)

$$
\begin{aligned}
& \Lambda\left(s, 1_{\mathfrak{f}} \lambda_{n} v\right)=f^{s / 2} \Lambda\left(s, \lambda_{n} v\right)\left\{1-\left(\lambda_{n} v\right)(\mathfrak{f}) f^{-s}\right\} \\
& =i^{-k(v)} f^{s / 2}\left(\lambda_{n} v\right)(\delta) \Lambda\left(1-s, \bar{\lambda}_{n} v\right)\left\{1-\left(\lambda_{n} v\right)(\mathfrak{T}) f^{-s}\right\} \\
& =i^{-k(v)} f^{s / 2}\left(\lambda_{n} v\right)(\delta) \Lambda\left(1-s, \bar{\lambda}_{n} v\right) \\
& -i^{-k(v)} f^{-s / 2}\left(\lambda_{n} v\right)(\delta \tilde{\uparrow})\left\{\Lambda\left(1-s, 1_{\mathfrak{f}} \bar{\lambda}_{n} v\right)+\Lambda\left(1-s, 0_{\mathfrak{f}} \bar{\lambda}_{n} v\right)\right. \\
& =i^{-k(v)} G\left(1_{\mathfrak{f}}\right) f^{-1 / 2}\left(\lambda_{n} v\right)(\delta \tilde{\uparrow}) \Lambda\left(1-s, 1_{\mathfrak{f}} \bar{\lambda}_{n} v\right) \\
& +i^{-k(v)}(f-1) f^{-1 / 2}\left(\lambda_{n} v\right)(\delta \tilde{\dagger}) \Lambda\left(1-s, 0_{\uparrow} \bar{\lambda}_{n} v\right) \text {. }
\end{aligned}
$$

We note that $\Lambda\left(s, 1_{\mathrm{f}} v_{0}\right)$ has a pole at $s=1$ but is regular at $s=0$. For $\alpha \not \equiv 0(\tilde{f})$ we have 


$$
\sum_{\chi} \chi(\alpha)= \begin{cases}\left(\lambda_{n} v\right)\left(\varepsilon^{-1}\right)(f-1) / e^{\prime}(\mathfrak{f}) & \text { if } \alpha \equiv \varepsilon(\mathfrak{F}) \text { for some } \varepsilon \in E, \\ 0 & \text { otherwise, }\end{cases}
$$

where $\chi$ runs over characters $\bmod \mathfrak{f}$ such that $\chi \lambda_{n} v$ is an ideal character $\bmod \mathfrak{f}$. By (3.2), (3.5) and (3.6),

$$
\begin{aligned}
& \frac{f-1}{e(\mathfrak{f}) e^{\prime}(\mathfrak{f})} P\left(s, \alpha \bmod \mathfrak{F}, \lambda_{n} v\right)=\sum_{\chi} \bar{\chi}(\alpha) \Lambda\left(s, \chi \lambda_{n} v\right) \\
& =i^{-k(v)} \sum_{z} \bar{\chi}(\alpha) G(\chi) f^{-1 / 2}\left(\lambda_{n} v\right)(\delta \tilde{\mp}) \Lambda\left(1-s, \chi \lambda_{n} v\right)
\end{aligned}
$$

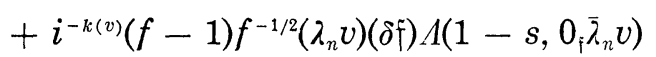

$$
\begin{aligned}
& =i^{-k(v)} f^{-1 / 2}\left(\lambda_{n} v\right)(\delta 千) \pi^{s-1}(D f)^{(1-s) / 2} \gamma\left(1-s ; \bar{\lambda}_{n} v\right)(f-1) \\
& \times\left\{e^{\prime}(\mathfrak{f})^{-1} \sum_{\substack{(c) \\
(c, \mathfrak{f})=1}}\left(\bar{\lambda}_{n} v\right)(c)|N(c)|^{s-1} \sum_{\varepsilon \in E / E \mathfrak{f}}\left(\bar{\lambda}_{n} v\right)(\varepsilon) \exp \left(2 \pi i S\left(\frac{\varepsilon \alpha^{c}}{\delta \tilde{f}}\right)\right)\right.
\end{aligned}
$$

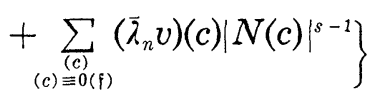

$$
\begin{aligned}
& =i^{-k(v)} \frac{f-1}{e(\mathfrak{f}) e^{\prime}(\mathfrak{f})} Q\left(1-s, \alpha \bmod \mathfrak{f}, \bar{\lambda}_{n} v\right) ;
\end{aligned}
$$

$P\left(s, \bmod \mathfrak{F}, \lambda_{n} v\right)(\alpha \neq \equiv(\mathfrak{F}))$ is analytically continued in the whole $s$-plane except a pole at $s=1$ in case of $\lambda_{n} v=v$; and

$$
\operatorname{Res}_{s=1} P\left(s, \alpha \bmod \mathfrak{f}, v_{0}\right)=4 f^{-1 / 2} \log \eta_{\mathfrak{f}} .
$$

Now our Lemma is proved.

\section{REFERENCES}

[1] E. Hecke, Über die Konstruktion der Klassenkörper reeller quadratischer Körper mit Hilfe von automorphen Funktionen, Math. Werke, Göttingen V Vandenhoeck \& Ruprecht, 1959, 64-68.

[2] - Eine neue Art von Zetafunktionen und ihre Beziehungen zur Verteilung der Primzahlen, Math. Werke, Göttingen•Vandenhoeck \& Ruprecht, 1959, 215-234.

[ 3 ] - Analytische Funktionen und algebraische Zahlen, I, II, Math. Werke Göttingen. Vandenhoeck \& Ruprecht, 1959, 336-360, 381-404.

[4] S. Konno, On Kronecker's limit formula in a totally imaginary quadratic field over a totally real algebraic number field, J. Math. Soc. Japan, 17 (1965), 411-424.

[5] C. L. Siegel, Über die Fourierschen Koeffizienten von Modulformen, Göttingen Nachr. Akad. Wiss., 1970, 15-56.

[6] A. Weil, Elliptic functions according to Eisenstein and Kronecker, Spri!gerVerlag-Berlin-Heidelberg-New York (1976).

\section{Department of Mathematics}

Ryukyu University

Okinawa, Japan 